\title{
I beta-bloccanti nella terapia dello scompenso cardiaco: revisione della letteratura
}

\author{
Gian Paolo Cioccia ', Carlo De Collibus ${ }^{2}$, Massimo Piccioni ${ }^{3}$
}

1 Dirigente medico I livello Coordinamento Generale Medico legale INPS. Docente Scuola Specializzazione in Medicina Legale Università "La Sapienza", Roma

2 Dirigente medico I livello Coordinamento Generale Medico legale INPS. Cardiologo c/o Centro Medico Legale Polispecialistico del Coordinamento Generale Medico Legale INPS

3 Coordinatore Generale Medico Legale INPS. Docente Scuola Specializzazione in Medicina Legale Università Cattolica Roma

\begin{abstract}
The incidence of medical malpractice litigation is increasing. Most of errors can be defined as "medication errors", that is to say inappropriate use and prescription of drugs. For this reason it is essential that physicians, and especially those who practices as specialists, know in-depth the effects of drugs that they prescribe. In cardiology, one important field is the risk of inappropriate prescription of beta blockers in the treatment of heart failure. Aim of this paper is to review the status of beta blockers prescription, searching for information concerning their inappropriate use (underuse or misuse). The research was made searching the most well-known juridical and medical databases according to these keywords: "beta blockers", "inappropriate use" and "heart failure".

The aim is to awake cardiologists in all the risks related to errors in this therapy, to meet the right standard of care and to avoid clinical risks.
\end{abstract}

Keywords: beta blockers, heart failure, inappropriate use

Beta blockers in heart failure: a literature review.

Pratica Medica \& Aspetti Legali 2008; 2(3): 119-123

\section{INTRODUZIONE}

La problematica del contenzioso per presunta malpractice da parte dei medici ha oramai assunto dimensioni preoccupanti. È quindi particolarmente importante che il medico, e ancor di più lo specialista, conoscano non soltanto le "dinamiche medico-legali" che sottendono alla responsabilità professionale, ma che siano sempre aggiornati sugli effetti dei farmaci che quotidianamente prescrivono.

Proprio in tale ottica abbiamo attuato una ricerca su casi di malpractice relativi all'errato uso dei beta-bloccanti nella terapia dello scompenso cardiaco.

La quantità di materiale reperito è stata enorme, ma non sempre rispecchiava gli standard di validi- tà scientifica. Per tale motivo abbiamo selezionato i dati provenienti da fonti scientificamente e giuridicamente accreditate quali: il repertorio del Foro italiano 1981-2006, Juris Data, Cicerone, Dottrina, Infoleges, Diritto e il sistema di ricerca Italgiureweb sul sito internet www.cortedicassazione.it.

Da questa ricerca analitica è emersa, a tutt'oggi, l'assenza di sentenze e/o casistica relative al nostro specifico argomento.

Conseguentemente abbiamo rivolto la nostra ricerca ad un ambito più "clinico" consultando i siti in rete più accreditati, quali PubMed, Teoma, Google Scholar, Univadis e Scirus con le parole chiave "beta blockers", "heart failure" e "inappropriate use" al fine di evidenziare, dai numerosi studi clinici trovati e selezionati, "linee guida" che possano orientare il medico cardiologo nella più corretta gestione farmacologica del paziente, limitando- 
ne così il rischio di esposizione a comportamenti potenzialmente censurabili.

La nostra ricerca vuole rappresentare uno spunto per eventuali più specifici approfondimenti che chi lo ritenesse opportuno potrà attuare seguendo i riferimenti bibliografici riportati per ogni lavoro vagliato.

\section{RISULTATI DELLA RICERCA}

Fernandes et al. [1] hanno condotto uno studio mirato alla valutazione dell'incidenza dei beta-bloccanti sulla mortalità e il loro effetto sull'apparato cardiovascolare entro un anno dal trattamento, in concomitanza con una delle seguenti condizioni cardiovascolari:

- post-infarto;

- scompenso cardiaco;

- stroke;

- angina;

- altre malattie ischemiche;

- ipertensione essenziale;

- shock cardiogeno;

- malattia dell'endocardio;

- disturbi della conduzione o cardiomiopatia.

Da questo studio emerge che l'appropriata prescrizione di beta-bloccanti per la prevenzione secondaria dopo un infarto miocardico è associata al miglioramento della sopravvivenza in una popolazione di età inferiore a 65 anni. L'analisi evidenzia che i casi di prescrizione inappropriata dei betabloccanti come agente di prevenzione secondaria in pazienti post-infartuati si riferiscono essenzial-

\begin{tabular}{|ll|}
\hline $\begin{array}{l}\text { Beta-bloccanti } \\
\text { non selettivi }\end{array}$ & Pindololo \\
& Propranololo \\
& Timololo \\
& Sotalolo \\
& Nadololo \\
\hline $\begin{array}{l}\text { Beta-bloccanti selettivi } \\
\text { non associati }\end{array}$ & Metoprololo \\
& Atenololo \\
& Acebutolo \\
& Betaxolo \\
& Bisoprololo \\
& Celiprololo \\
& Esmololo \\
\hline $\begin{array}{l}\text { Beta-bloccanti che agiscono } \\
\text { anche sui recettori } \\
\text { alfa-adrenergici }\end{array}$ & Labetalolo \\
\hline
\end{tabular}

Tabella I. Principali beta-bloccanti mente a sotto-utilizzo (errore omissivo) oppure uso errato (errore commissivo).

L'uso errato di beta-bloccanti non è limitato solo alle patologie cardiovascolari. Ad esempio Anis et al. [2] hanno verificato che i pazienti asmatici sottoposti ad un trattamento farmacologico inadeguato (consistente in un impiego eccessivo di beta-bloccanti e in un uso carente di corticosteroidi) hanno subìto più frequenti ricoveri in ospedale e hanno richiesto al dipartimento di emergenza accessi più numerosi di quelli dei pazienti che hanno assunto un normale dosaggio di farmaco.

La terapia con beta-bloccanti (Tabella I) attualmente è raccomandata come strategia di prima linea per lo scompenso cardiaco. Tuttavia, le prescrizioni di questi farmaci risultano molto inferiori a quelle degli ACE-inibitori.

Secondo Hery et al. [3] questo basso impiego può essere spiegato come una conseguenza dei potenziali effetti collaterali dei beta-bloccanti quali bradicardia, ipotensione e specialmente scompenso cardiaco acuto.

I dati clinici potrebbero evidenziare facilmente pazienti ad alto rischio per ipotensione arteriosa o bradicardia, ma non pazienti ad alto rischio di insufficienza cardiaca indotta dal trattamento con beta-bloccanti. La determinazione del BNP (Peptide Natriuretico di tipo $B)$ può rivelarsi molto utile in questo contesto. Il riscontro di un valore di BNP superiore a $490 \mathrm{pg} / \mathrm{ml}$ precedente all'introduzione del trattamento con beta-bloccanti consente di identificare pazienti ad alto rischio di insufficienza cardiaca da terapia con beta-bloccanti con una sensibilità del $\mathbf{9 0 \%}$; un valore di BNP superiore a $1.000 \mathrm{pg} / \mathrm{ml}$ identifica i pazienti con il $40 \%$ di rischio di insufficienza cardiaca acuta dopo l'inizio del trattamento con beta-bloccanti o l'aumento della dose di questi ultimi. Pertanto deve essere osservata molta cautela nell'inizio del trattamento con carvedilolo o nell'aumento del dosaggio di quest'ultimo nei soggetti con elevati valori di BNP.

Le linee guida dell'American College of Cardiology e dell'American Heart Association Task Force [4] hanno fortemente raccomandato l'uso dei beta-bloccanti nello scompenso cardiaco, ma hanno messo in rilievo i comuni effetti sfavorevoli cardiovascolari comprendenti l'aggravamento dello scompenso cardiaco, vertigini, bradicardia e affaticamento. Esse inoltre raccomandano l'uso dei beta-bloccanti in tutti i pazienti con disfunzione ventricolare sinistra stabile sintomatica.

Per quanto riguarda pazienti con disfunzione ventricolare sinistra asintomatica, le linee guida raccomandano l'uso dei beta-bloccanti nei pazienti con recente infarto miocardico senza riguardo alla frazione di eiezione del ventricolo sinistro e nei pazienti con una ridotta frazione di eiezione ven- 
tricolare sinistra indipendentemente dal fatto che abbiano o meno subito un infarto miocardico. Secondo le linee guida europee del 2001 [5] i betabloccanti sono indicati in tutti casi di scompenso cardiaco lieve, medio e severo in condizioni di stabilità clinica dovuto a cardiomiopatia ischemica 0 non ischemica con ridotta frazione di eiezione $\mathrm{e}$ in classe NYHA II-IV (Tabella II) in terapia con ACE-inibitori e diuretici salvo controindicazioni (livello di evidenza A); i beta-bloccanti sono indicati a lungo termine in caso di disfunzione ventricolare sinistra anche asintomatica dopo infarto miocardico.

Tra i criteri di esclusione impiegati nei grandi trial sull'impiego dei beta-bloccanti nello scompenso cardiaco si annovera la concomitanza di patologie quali:

- arteriopatie periferiche limitanti l'esercizio;

- cuore polmonare;

- broncopneumopatia cronica ostruttiva (BPCO) necessitante terapia broncodilatatrice orale o terapia steroidea;

- diabete mellito insulino-dipendente instabile richiedente frequenti ospedalizzazioni;

- storia di reazioni avverse agli alfa-bloccanti o ai beta-bloccanti [6];

- asma [7];

- qualsiasi condizione oltre insufficienza cardiaca che possa limitare l'esercizio fisico, quali angina, malattia vascolare periferica, malattia polmonare [8];

- cronica limitazione del flusso d'aria con evidenza del $20 \%$ o maggiore reversibilità della ostruzione del flusso d'aria in risposta alla inalazione di salbutamolo [9].

In particolare $\mathrm{i}$ beta-bloccanti dovrebbero essere evitati solo in presenza di fenomeni vasospastici, di dolori a riposo in pazienti con arteriopatia di grado severo e di lesioni trofiche cutanee; non esiste alcuna controindicazione assoluta nei pazienti in trattamento insulinico, ma particolare cautela deve essere riservata a pazienti con difficile controllo dei valori glicemici e/o in trattamento con antidiabetici orali a lunga durata di azione per la possibilità di prolungate ipoglicemie paucisintomatiche. Lasma bronchiale rappresenta una controindicazione assoluta. Nell'ambito delle controindicazioni assolute sono poi da annoverarsi la BPCO di grado moderato-severo (stadio II-III con $\mathrm{FEV}_{1}<$ al $50 \%$ del predetto), i pazienti in trattamento cronico con broncodilatatori, la BPCO con presenza di reversibilità della bronco-ostruzione indotta dall'inalazione di salbutamolo $\leq 20 \%$ del valore del $\mathrm{FEV}_{1}$ basale (oppure $12 \%$ del predetto) e almeno $>$ di $200 \mathrm{ml}[9]$.

Nei primi giorni di trattamento la frazione di eiezione del ventricolo sinistro va incontro a una lieve riduzione nell'ordine di 1-2 punti percentuali, ma, dopo almeno un mese di terapia con beta-

\begin{tabular}{|cl|}
\hline Classe & \multicolumn{1}{c|}{ Descrizione } \\
\hline I & Asintomatica \\
\hline II & Sintomi con sforzo moderato \\
\hline III & Sintomi con sforzo minimo \\
\hline IV & Sintomi a riposo \\
\hline
\end{tabular}

Tabella II. Classificazione della New York Heart Association (NYHA) della capacità funzionale dopo scompenso cardiaco

bloccanti, la risposta, sotto il profilo strettamente emodinamico, è mediamente caratterizzata da aumento della gittata sistolica, riduzione delle pressioni medie atriali e della pressione telediastolica del ventricolo sinistro, aumento dell'indice di lavoro sistolico del ventricolo sinistro.

La tollerabilità acuta del beta-bloccante è probabilmente migliore per carvedilolo grazie alle associate proprietà di vasodilatazione periferica che permettono di antagonizzare l'iniziale riduzione acuta dell'inotropismo attraverso la riduzione del post-carico; dunque i beta-bloccanti non sono necessariamente inotropo-negativi; nello scompenso cardiaco il loro impiego cronico si traduce in un effetto inotropo-positivo [10].

Obiettivo dello studio condotto da Ko et al. [11] è stato quello di quantificare il rischio di comuni e potenzialmente gravi effetti collaterali della terapia con beta-bloccanti in pazienti in scompenso cardiaco con disfunzione sistolica.

In base all'analisi di trial reperiti mediante ricerca elettronica sul database PubMed (1966-2002), l'uso dei beta-bloccanti in soggetti con scompenso cardiaco è stato associato a un rischio aumentato di ipotensione e vertigini; inoltre gli incrementi assoluti del rischio predetto sono stati modesti e i pazienti raramente hanno sospeso il trattamento a causa dei loro sintomi. È stato rilevato che i betabloccanti hanno effetti isotropo-negativi che potrebbero causare una perdita di compenso cardiaco in soggetti con insufficienza cardiaca e disfunzione ventricolare. L'ipotensione nei soggetti con insufficienza cardiaca è un elemento di rilievo dal momento che può peggiorare la perfusione degli organi più importanti.

Le vertigini possono accompagnare l'ipotensione e interferire con la vita quotidiana.

I beta-bloccanti abbassano la pressione arteriosa attraverso svariati meccanismi comprendenti la riduzione della gittata cardiaca e del tono simpatico, come pure la vasodilatazione per i beta-bloccanti con concomitante attività alfa-bloccante.

Una marcata bradicardia è fonte di preoccupazione nei pazienti con insufficienza cardiaca, in quanto può ridurre la gittata cardiaca e causare scompenso clinico. Il miglioramento della sopravvivenza ottenuto mediante trattamento con beta-bloccanti può essere associato a un peggioramento 
della qualità di vita dovuto all'affaticamento, riconosciuto un effetto sfavorevole del trattamento con beta-bloccanti, ipoteticamente correlato alla riduzione della gittata cardiaca e agli effetti sul sistema nervoso centrale.

Gronda et al. [12] hanno rilevato che l'effetto del beta-bloccante nei pazienti con scompenso cardiaco presenta un andamento bifasico: un possibile iniziale deterioramento clinico determinato dall'ostacolo ai meccanismi di compenso messi in funzione dall'organismo seguito nei mesi successivi da un più prolungato beneficio in termini clinici e strumentali.

Per ridurre l'iniziale deterioramento emodinamico è importante iniziare con dosi molto basse del farmaco, proseguendo con aumenti lenti e progressivi del dosaggio nelle settimane successive al fine di consentire il ristabilirsi di un nuovo equilibrio neuroumorale. Anche con tale accorgimento può verificarsi un peggioramento clinico con incidenza proporzionale alla severità del quadro clinico iniziale.

Alcuni studi hanno verificato la tollerabilità dei beta-bloccanti in relazione alle classi funzionali NYHA. Macdonald et al. [13] hanno riportato un'incidenza di intolleranza pari al $10 \%$ nei soggetti in classe II e III e pari al $22 \%$ in classe IV.

In un secondo studio [14], l'incidenza di intolleranza nella IV classe funzionale saliva al $37 \%$; peraltro nella maggior parte dei casi un rallentamento nell'incremento del farmaco e un attento aggiustamento di dosaggio dei farmaci associati risolve gli effetti indesiderati.

Analogamente Macdonald, nello studio sopra citato [13], condotto per verificare la tollerabilità e l'efficacia di carvedilolo in pazienti in classe funzionale NYHA IV, ha rilevato che i pazienti con scompenso cardiaco cronico appartenenti a questa classe funzionale sviluppano, più facilmente di pazienti in classe funzionale inferiore, effetti sfavorevoli quali peggioramento dello scompenso cardiaco, bradicardia sintomatica, ipotensione arteriosa e affaticamento, ma dimostrano più facilmente miglioramento sintomatologico a lungo termine.

In particolare, in pazienti affetti da scompenso cardiaco in classe funzionale NYHA IV con iposodiemia sierica $\left(\mathrm{Na}^{+}\right.$sierico $\left.<137.000 \mathrm{mml}\right)$ all'inizio del trattamento con carvedilolo sono andati incontro a un effetto sfavorevole più frequentemente dei pazienti in classe funzionale NYHA IV che avevano un normale valore di sodio sierico all'inizio del trattamento.

Sackner-Bernstein et al. [15] hanno inoltre verificato che l'iponatremia è il più potente predittore di eventi sfavorevoli dopo l'inizio del trattamento con carvedilolo in pazienti con scompenso cardiaco da moderato a severo: è stato dimostrato che l'iponatremia è strettamente correlata all'attivazione neuroumorale nello scompenso cardiaco, in particolare del sistema renina-angiotensina, e che è un fattore primitivo indipendente di mortalità nei pazienti con scompenso cardiaco severo.

È stato inoltre rilevato che i pazienti iponatriemici sono più facilmente ipotesi ed evidenziano un più facile peggioramento della perfusione periferica e renale rispetto ai pazienti con scompenso cardiaco in classe NYHA IV con normale sodio sierico.

In una review sull'uso di bisoprololo nello scompenso cardiaco cronico condotta da McGavin et al. [16] è stato verificato che bisoprololo è generalmente ben tollerato nei pazienti con scompenso cardiaco cronico.

Negli studi CIBIS e CIBIS II [7] è stata confrontata l'incidenza di effetti collaterali nel trattamento dello scompenso cardiaco cronico con bisoprololo rispetto a placebo; ne è emerso che:

- sono più frequenti, nel caso di bisoprololo, vertigini, bradicardia, ipotensione, affaticamento;

- sono meno frequenti, nel caso di bisoprololo, il peggioramento dello scompenso cardiaco, dispnea o tachicardia.

Analogamente, come rilevato da Deedswania et al. [17], bradicardia, ipotensione, vertigini, affaticamento e dispnea hanno motivato l'interruzione dell'assunzione di metoprololo a rilascio controllato-prolungato nello studio MERIT-HF condotto in pazienti di età $>65$ anni con scompenso cardiaco dovuto a disfunzione sistolica ventricolare sinistra.

Ko DT et al. [11], previa ricerca condotta su PubMed dal 1966 al 2002, hanno concluso che i betabloccanti hanno aumentato ipotensione arteriosa, vertigini e bradicardia nella popolazione con scompenso cardiaco e disfunzione sistolica, ma gli incrementi assoluti del rischio sono stati modesti. Bisoprololo è stato approvato per il trattamento di pazienti con scompenso cardiaco stabile cronico moderato-severo con ridotta funzione sistolica ventricolare sinistra (LVEF $\leq 35 \%$ confermato dall'ecocardiografia) in numerose Nazioni, compresi la maggior parte dei Paesi europei e latino-americani e svariati Paesi del sud-est asiatico. Questo farmaco, peraltro, è controindicato nei pazienti con scompenso cardiaco acuto che richiede terapia endovenosa, in shock cardiogeno, con un blocco atrio-ventricolare di II-III grado (senza pacemaker), con malattia del nodo del seno, con blocco seno-atriale, con bradicardia (frequenza cardiaca inferiore a 60 battiti al minuto precedentemente alla terapia) o con ipotensione (pressione arteriosa sistolica inferiore a $100 \mathrm{mmHg}$ ), in pazienti con asma bronchiale severa o con severa malattia polmonare cronico-ostruttiva, malattia occlusiva arteriosa periferica all'ultimo stadio, sindrome di Raynaud, feocromocitoma non trattato o acidosi metabolica. 


\section{CONCLUSIONI}

Alla luce di quanto emerso dalla nostra ricerca, è confermata la necessità di una attenta valutazione preliminare del paziente, volta a una prescrizione terapeutica personalizzata. Particolare cautela, quindi, nella conoscenza e valutazione delle controindicazioni all'uso dei beta-bloccanti che dovranno essere prescritti peculiarmente nei casi di scompenso cardiaco lieve-medio-severo in condizioni di stabilità clinica da cardiomiopatia ischemica e/o non ischemica con ridotta frazione di eiezione in classe NYHA II-IV, in terapia con ACE-inibitori e diuretici salvo specifiche controindicazioni precedentemente descritte. Inoltre i beta-bloc- canti sono indicati a lungo termine in caso di disfunzione ventricolare sinistra anche asintomatica dopo infarto miocardico.

Si ribadisce l'importanza delle linee guida intese come raccomandazioni di comportamento clinico, prodotte attraverso un processo sistematico allo scopo di coadiuvare medici e pazienti nel decidere quali siano le modalità di assistenza più appropriate in specifiche circostanze cliniche, quindi, valido supporto per i Sanitari non solo nella promozione delle conoscenze, nella riduzione della variabilità dei comportamenti, nel trasferimento dei risultati della ricerca alla pratica clinica quotidiana, nel controllo dei costi sanitari ma anche quale strumento di indubbia tutela del paziente e del medico.

\section{BIBLIOGRAFIA}

1. Fernandes AW, Madhavan SS, Amonkar MM. Evaluating the effect on patient outcomes of appropriate and inappropriate use of beta-blockers as secondary prevention after myocardial infarction in a medicaid population. Clin Ther 2005; 27: 630- 45

2. Anis AH, Lynd LD, Wang XH, King G, Spinelli JJ, Fitzgerald M et al. Double trouble: impact of inappropriate use of asthma medication on the use of health care resources. CMAJ 2001; 164: 625-31

3. Hery E, Jourdain P, Funck F, Bellorini M, Loiret J, Thebault B et al. Prediction of intolerance to beta blocker therapy in chronic heart failure patients using BNP. Annales de Cardiologie et d'Angéiologie 2004; 53: 298-304

4. Hunt SA, Baker DW, Chin MH, Cinquegrani MP, Feldman AM, Francis GS et al. ACC/AHA guidelines for the evaluation and management of chronic heart failure in the adult: executive summary: a report of the american college of cardiology/american heart association task force on practice guidelines (committee to revise the 1995 guidelines for the evaluation and management of heart failure) developed in collaboration with the international society for heart and lung transplantation endorsed by the Heart Failure Society of America. J Am Coll Cardiol 2001; 38: 2101-13

5. Task Force Report Guidelines for the diagnosis and treatment of chronic heart failure. Eur Heart J 2001; 22: 1527-1560

6. Bristow MR, Gilbert EM, Abraham WT, Adams KF, Fowler MB, Hershberger RE et al. Carvedilol produces dose-related improvements in left ventricular function and survival in subjects with chronic heart failure. MOCHA Investigators. Circulation 1996; 94: 2807-16

7. Simon T, Mary-Krause M, Funck-Brentano C, Lechat P, Jaillon P. Bisoprolol dose-response relationship in patients with congestive heart failure: a subgroup analysis in the cardiac insufficiency bisoprolol study (CIBIS II). Eur Heart J 2003; 24: 552-9

8. Packer M, Colucci WS, Sackner-Bernstein JD, Liang C, Goldscher DA, Freeman I et al for the PRECISE Study Group. Double-blind, placebo-controlled study of the effects of carvedilol in patients with moderate to severe heart failure: the PRECISE trial. Circulation 1996; 94: 2793-9

9. Pozzi R. True and presumed contraindications of beta blockers. Peripheral vascular disease, diabetes mellitus, chronic bronchopneumopathy. Ital Heart J Suppl 2000; 1: 1031-7

10. Rapezzi C, Branzi A. Critical analysis of beta blockers in heart failure: certainty and incompleteness. Ital Heart J Suppl 2000; 1 : 1003-10

11. Ko DT, Hebert PR, Coffey CS, Curtis JP, Foody JM, Sedrakyan A et al. Adverse effects of beta-blocker therapy for patients with heart failure: a quantitative overview of randomized trials. Arch Intern Med 2004; 164: 1389-94

12. Gronda E, Bologna A, Mangiavacchi M, Andreuzzi B. Treatment with beta blockers in advanced heart failure. Ital Heart J Suppl 2000; $1: 1011-8$

13. Macdonald PS, Keogh AM, Aboyoun C, Lund M, Amor R, McCaffrey D. Impact of concurrent amiodarone treatment on the tolerability and efficacy of carvedilol in patients with chronic heart failure. Heart 1999; 82: 589-93

14. Krum H, Sackner-Bernstein JD, Goldsmith RL, Kukin ML, Schwartz B, Penn J. Double-blind, placebo-controlled study of the long term efficacy of carvedilol in patients with severe chronic heart failure. Circulation 1995; 92: 1499-506

15. Sackner-Bernstein J, Krum H, Goldsmith R, et al. Should worsening heart failure early after initiatin of beta-blocker therapy for chronic heart failure preclude long-term treatment. Circulation 1995; 92: I-395

16. Deedwania PC, Gottlieb S, Ghali JK, Waagstein F, Wikstrand JC for the MERIT-HF Study Group. Efficacy, safety and tolerability of beta-adrenergic blockade with metoprolol CR/XL in elderly patients with heart failure. Eur Heart J 2004; 25: 1300-9

17. McGavin JK, Keating GM. Bisoprolol: a review of its use in chronic heart failure. Drugs 2002; 62: 2677-96 